Relations industrielles

Industrial Relations

\title{
Compulsory Arbitration - The Australian Way? Industrial Relations Society of Australia, Papers presented at the 1986 National Convention, Adelaide, September 1986, 192 pp.
}

\section{Pierre Verge}

\section{Volume 42, numéro 4, 1987}

URI : https://id.erudit.org/iderudit/050375ar

DOI : https://doi.org/10.7202/050375ar

Aller au sommaire du numéro

Éditeur(s)

Département des relations industrielles de l'Université Laval

ISSN

0034-379X (imprimé)

1703-8138 (numérique)

Découvrir la revue

Citer ce compte rendu

Verge, P. (1987). Compte rendu de [Compulsory Arbitration - The Australian Way? Industrial Relations Society of Australia, Papers presented at the 1986 National Convention, Adelaide, September 1986, 192 pp.] Relations industrielles / Industrial Relations, 42(4), 886-887. https://doi.org/10.7202/050375ar

Tous droits réservés (C) Département des relations industrielles de l'Université Laval, 1987
Ce document est protégé par la loi sur le droit d'auteur. L’utilisation des services d’Érudit (y compris la reproduction) est assujettie à sa politique d'utilisation que vous pouvez consulter en ligne.

https://apropos.erudit.org/fr/usagers/politique-dutilisation/ 
totalitaires (l'Italie, dès 1922, l'Allemagne à compter de 1933 et la France pétainiste). Le conflit mondial lui-même allait préparer des affirmations plus accentuées de droits et de libertés de l'Homme, et leurs répercussions sur le travail.

Toutes ces fresques détaillées en mouvement, en plus de faire ressortir les racines et les grands courants d'une bonne partie du droit du travail occidental, constituent de surcroît, un modèle d'analyse susceptible de transpositions, comme plusieurs des éléments de ce droit du travail lui-même... D'où leur importance singulière.

Pierre VERGE

Université Laval

Compulsory Arbitration - The Australian Way? by Industrial Relations Society of Australia, ed., Papers presented at the 1986 National Convention, Adelaide, September 1986, $192 \mathrm{pp}$.

Il y a lieu de signaler la parution de cet ensemble de huit textes relatifs au système australien de conciliation et d'arbitrage des conflits du travail, du moins à l'avantage des spécialistes qui possèdent déjà une connaissance du sujet.

Ces différentes études, en effet, s'adressent primordialement à un public local; elles renferment des appréciations, souvent fort détaillées, du fonctionnement du système australien de rapports collectifs du travail, dont les origines remontent à 1904. Le recueil, compte tenu de son orientation, ne contient cependant aucune présentation générale et méthodique de ce système, au détriment du lecteur étranger qui ne serait pas déjà averti de la question.

Le rapport Hancock (Committee of Review into Australian Industrial Relations Law and Systems, Report, AGPS, Canberra 1985) est l'occasion de cet examen de conscience de la part d'acteurs et d'observateurs nationaux. Ses recommandations sont révisionnistes; il s'agit, manifestement, d'améliorer une formule idiosyncratique qui, tout compte fait, semble bien adaptée aux besoins du milieu qu'elle dessert. D'ailleurs, les seules vues ternes vont en ce sens. Le professeur Benjamin Aaron, de l'Université de Californie, après avoir brossé un tableau de certains grands modes occidentaux de solution des conflits du travail, n'en abstrait, en effet, que quelques éléments particuliers aux fins d'une éventuelle transplantation en sol australien. Ainsi, le système américain d'arbitrage volontaire des griefs de convention collective repose sur un système décentralisé de détermination collective du salaire, ce qui l'éloigne de la tradition d'Australie. Ses observations du fonctionnement de la négociation collective aux États-Unies, jointes à celles, plus générales, qu'il formule relativement aux systèmes d'Angleterre, de France et de la R.F.A., le conduisent au bilan suivant (p. 173):

«Thus, arguably, (the present Australian compulsory arbitration system) might be improved by strengtening private disputes - settlement arrangements, particularly at the entreprise level; by developing more carefully structured voluntary grievance and arbitration procedures; by placing even greater emphasis than at present on conciliation as an integral part of the process preceding compulsory arbitration; by introducing tripartitism into all disputes - settlement machinery; and by making the entire system less formal and less dominated by lawyers and judges (...)»

Parmi les textes proprement australiens, on notera en particulier celui du professeur Hancock (the Flinders University of South Australia), qui apporte le recul de la perspective historique, l'analyse juridique du professeur Brooks, (University of New South Wales), qui tente de déterminer jusqu'à quel point la constitution d'Australie permet au tribunal industriel fédéral 
de s'immiscer dans les prérogatives de la direction et, plus généralement, dans la gouverne de l'économie du pays, ainsi que les réflexions du juge Boulton, de la Australian Conciliation and Arbitration Commission, sur les rôles respectifs du volontariat et des sanctions dans le fonctionnement du système d'arbitrage obligatoire d'Australie.

En bref, selon le résumé d'un avocat du pays, «The Australian Way of Compulsory arbitration is to be preferred to other models, at least for the time being) (p. 192). Le lecteur canadien - celui, en particulier qui voudrait se préparer au congrès mondial de l'Association internationale des relations industrielles, qui aura lieu en Australie en 1992 - pourra, grâce à ces pages, trouver utile d'actualiser ses connaissances générales et préalables du système de rapports du travail qui prévaut en ce pays.

Pierre VERGE

Université Laval

Young Adult in the Labour Market, par D.N. Ashton and J.J. Maguire, Research Paper no. 55, Leicester, University of Leicester, 1986, XIII, 163 pages.

Dans le cadre de son programme de recherche sur l'emploi et les relations industrielles, le ministère de l'Emploi de l'Angleterre a publié cette étude sur les jeunes de 18 à 24 ans, groupe dont l'incidence du chômage est très forte, comme c'est aussi le cas au Canada. Celle-ci a été réalisée par une équipe de recherche de l'Université de Leicester. Elle vise à mieux comprendre le comportement des jeunes sur le marché du travail en identifiant les facteurs qui affectent leur attitude à l'égard de l'emploi, de la mobilité et de la formation.

La théorie économique traditionnelle explique la plus forte incidence du chômage chez les jeunes principalement par des niveaux trop élevés de salaire par rapport à leur productivité et par des comportements d'instabilité sur le marché du travail. La plus forte instabilité résulterait de caractéristiques personnelles, soit une plus faible éthique du travail, soit une place normale d'adaptation au marché du travail par l'expérimentation.

À travers divers types d'emploi, cette thèse sur le chômage des jeunes conduit à des recommandations de politiques entre autres relatives au salaire minimum et à la formation pour améliorer leur productivité.

Une autre thèse, celle de la segmentation des marchés du travail, conduit à des politiques souvent différentes sinon de nature au moins d'application. Selon cette thèse, la plus forte incidence du chômage chez certains groupes démographiques n'est pas surtout le résultat de caractéristiques personnelles inhérentes à cette main-d'oeuvre, mais il est attribuable à des caractéristiques du marché du travail. Celui-ci serait segmenté à cause de pratiques d'embauche et de promotion, de normes institutionnelles et de perceptions des employeurs quant aux capacités de certains groupes démographiques d'occuper certaines filières d'emploi. Les segments du marché du travail auxquels les jeunes ont accès présentent des caractéristiques fort différentes du segment des emplois d'adultes. Ces caractéristiques affectent les comportements et les attitudes des jeunes adultes et expliqueraient leur chômage plus élevé.

Au Canada, les thèses de la segmentation ont surtout alimenté les réflexions sur le marché du travail féminin et ont conduit à des politiques d'action positive. 